\title{
The study of Mycoplasma pneumonia infection among children with respiratory tract infection in hospital in Chengdu from 2014 to 2020
}

lei zhang

University of Electronic Science and Technology of China

Meimei Lai

University of Electronic Science and Technology of China

Tao Ai ( $\square$ zhanglei78322@163.com )

University of Electronic Science and Technology of China

Huilling Liao

University of Electronic Science and Technology of China

Yijie Huang

University of Electronic Science and Technology of China

Ying Zhang

University of Electronic Science and Technology of China

Yanru Liu

University of Electronic Science and Technology of China

Li Wang

University of Electronic Science and Technology of China

Jie Hu

University of Electronic Science and Technology of China

\section{Research Article}

Keywords: Respiratory tract infection, Children, Mycoplasma pneumonia,(M. pneumonia)

Posted Date: January 21st, 2021

DOI: https://doi.org/10.21203/rs.3.rs-144312/v1

License: (c) (i) This work is licensed under a Creative Commons Attribution 4.0 International License.

Read Full License 


\section{Abstract}

Aim: The hospitalized children with Mycoplasma pneumonia (M. pneumonia) infection caused by respiratory tract infection in Chengdu were studied and analysis of the epidemiological characteristics was carried out to provide a theoretical basis for clinical diagnosis and treatment.

Method: 22882 hospitalized children with respiratory tract infections between January 2014 and December 2020 were collected M. pneumonia IgM antibody was detected by indirect immunofluorescence method and passive agglutination method. Demographic characteristics, clinical diagnose and laboratory data of these children were analyzed.retrospectively..

Result : The 4213 specimens with M. pneumonia were tested positive, the total positive rate was $18.41 \%$ (18.30\% in male and $22.72 \%$ in female). Higher positive rates were found in female children,Look from

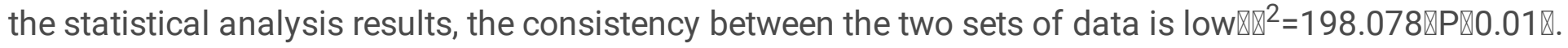
The results of different age patients with contrast different $M$. pneumonia infection degree were statistically significant $\triangle \mathrm{F}=162.7532 \square \mathrm{P} \unrhd 0.01 \rrbracket$,there was higher $M$. pneumonia positive rate in Preschoolers and school-age children ,33.98\% and 32.98\%, respectively. The incidence rate of M. pneumonia in 2017 and 2019 was significantly higher than average $\otimes F=538.95, P \llbracket 0.01$ ) The difference of incidence rate of $M$. pneumonia was not significant in different months in 2014, 2015 and 2020 ( $P>0.05)$. But the probability of $M$.pneumonia infection patients was much higher from April to May and September to October in2016,2017,2018 and 2019هP $₫ 0.05 \rrbracket$. There was no correlation about $M$. pneumonia infection with

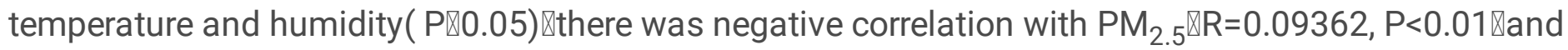
$\mathrm{PM}_{10} . \mathrm{QR}=0.1185, \mathrm{P}<0.01 \rrbracket$. There was no difference about constituent ratio of case of $M$. pneumonia infection between 2014 and 2019 ( $F=32.34, P>0.05)$. The Common respiratory diseases of M. pneumonia infection, bronchopneumonia accounts for the highest proportion, followed the exacerbation of asthma and severe pneumonia.There was significantly difference about constituent ratio of case of $M$. pneumonia infection between in 2020 and in other years $(F=159.35, P<0.01)$. The Common respiratory diseases of $M$. pneumonia infection, bronchopneumonia accounts for the highest proportion,followed the acute bronchitis and exacerbation of asthma.

Conclusion:The distribution and epidemiological trend of M. pneumonia in patients with respiratory tract infection showed the risk of inflammation was connected with the gender, age, year and month, no relationship with temperature and humidity in Chengdu..Higher M. pneumonia positive rate was shown in the children with bronchial pneumonia and exacerbation of asthma.The prevention measures which controlled the COVID-19 disease had effectively controlled the infection rate of M. pneumonia.

\section{Background}

Respiratory tract infection is one of common diseases among the children which could be caused by a board range of pathogens including viruses ,bacterial pathogens and atypical bacterial pathogens. Mycoplasma pneumonia is a common bacteral pathogen which is dominated by lower respiratory tract 
infections in children .M.pneumonia is one of the smallest prokaryotic cell type of microorganisms that lack of cell walls, highly polymorphic, and can pass the bacteria filter and grow on the inanimate medium. In recent years, the incidence of $M$. pneumonia infection rate has an increasing trend ${ }^{[1-3]}$.

There is different pathogen spectrum and epidemiological feature in different areas in the city. Up to date,there are fragmentary data about prevalence of M. pneumonia infection pediatric patients in Chengdu, Sichuan Province, China, including during the time which 2019 coronavirus diseases (COVID19) spreaded in China.The aim of this paper is mainly to understand the epidemiological distribution of M. pneumonia in hospitalized Children between January 2014and December 2020

Informed consent was obtained from a parent of patients. The retrosceptive studies was approved by the ethics committee of the Chengdu Women's and Children's Hospital ,SiChuan, China.

\section{Methods}

\section{Subject information}

- The study was conducted on22882 inpatients suffering from respiratory tract infection in Chengdu Women's and Children's Hospital, Chengdu, China. Diagnosis followed World Health Organization Criteria. Among the patients, 13600 children were male and the rest were female. Aged from 1month to 17years old. Ages were classified into subgroups corresponding to different physiological stages. infants group ( 1 month $₫ 1$ year old),toddlers group $(₫ 1 \otimes 3$ years old),preschoolers group ( $₫ 3 \otimes 6$ years old),school-age children group(7囚17 years old).Serums sample of every patient were separated from venous blood drawn and tested with pneumonia IgM and antibody. The case was defined with $M$. pneumonia infection, which $M$. pneumonia IgM was positive and antibody $\geq 1: 160 .{ }^{[4]}$ Demographic features of patients,clinical diagnose and laboratory data were analyzed . To explore whether there was an association with gender, age, month, year, $\mathrm{PM}_{2.5}, \mathrm{PM}_{10}$, temperature, humidity (data was kindly provided by Chengdu Meteorological Service】and the aetiological organism,.all methods were carried out in accordance with relevant guidelines and regulations

\section{Blood sampling}

- 22882 samples of venous blood were drawn from each child. The samples were centrifuged at $2000 \mathrm{~g}$ for $10 \mathrm{~min}$ at $4^{\circ} \mathrm{C}$. Serum was separated firstly and then stored at $-20^{\circ} \mathrm{C}$ until it was assayed with the pneumonia IgM and antibody test.

\section{Pneumonia IgM test (Indirect immunity)and antibody positive titer test .}

First at 1:1 ratio in the sample with phosphate buffer salt (PBS), get after dilution of the sample, then drip in the samples of human immunoglobulin adsorbent, and the sample in $37^{\circ} \mathrm{C}$ slide drilling stand for 90 minutes, to join in the first samples of two kinds of fluorescent auxiliary agent in PBS, observing the existence of IgM antibody in the samples, and placed at $37^{\circ} \mathrm{C}$ environment, wait 30 minutes later, the two 
samples are recorded with a fluorescent microscope imaging of imaging signal, ( Zeiss, Germany)..IgM antibodies against $M$. pneumonia in serum were detected using a passive agglutination kid (Fujirebio, Japan) based on the manufacturer's instructions.

\section{Statistical analysis}

Categorical variables were given as numbers with percentages and a value of $p<0.05$ was considered to be statistically significant when compared with $\mathrm{X}_{2}$-test. Meanwhile, the risk factors of $\boldsymbol{M}$. pneumonia infection were assessed by using binary logistic regression. Associations were quantified with odds ratios (OR) and their $95 \%$ confidence intervals $(\mathrm{Cl})$. Analyses were conducted using the Statistical Package for the Social Sciences for Windows (Spss, version11.0)

\section{Results}

The positive rates of $M$. pneumonia infection in different genders .

Of 22882samples tested, 4213 samples were positive for a positive percentage of $18.41 \%$. 2104 samples were positive for a positive percentage of $18.30 \%$ in male, while 2109 samples for $22.72 \%$ in female. ( $\mathbb{~}$ $2=198.078 \otimes P \otimes 0.01 \rrbracket$

\section{The positive rates of $M$. pneumonia infection in different age groups}

As a whole to sum up, the positive percentage of M. pneumonia infection was $4.24 \%$ in infants, $26.92 \%$ in toddlers, $33.98 \%$ in preschoolers and $32.98 \%$ in school-age children. As listed in Table 1, the positive percentage increased with the age of the patients $\mathbb{F}=162.7532 \square \mathrm{P} \otimes 0.01 \rrbracket($ Table 1$)$

Table 1: $\boldsymbol{M}$. pneumonia positive rates among different age groups

\begin{tabular}{|lllll|}
\hline & Infants & Toddlers & Preschoolers & School-age children \\
\hline Positive sample & 405 & 2708 & 753 & 347 \\
\hline Total sample & 9555 & 10059 & 2216 & 1052 \\
\hline Positive percentage(\%) & 4.24 & 26.92 & 33.98 & 32.98 \\
\hline
\end{tabular}

$\triangle \mathrm{F}=162.7532 \mathrm{P} \otimes 0.01 \rrbracket$

\section{Annual incidence and seasonal incidence of $M$. pneumonia infection}

Each year, the number of patients with $M$. pneumonia infection was significant difference $(F=538.95$ $\mathrm{P}<0.01)$. It had a relatively lower incidence of $M$. pneumonia infection in $2014(13.14 \%), 2015(10.47 \%)$ and 2020(17.98\%). The incidence of M. pneumonia infection were significantly higher in 2017 and 2019 ,respectively $26.59 \%$ and $28.18 \%$, among them, The incidence of $M$. pneumonia infection are mainly concentrated in 4, 5, 9, 10, four months in $2016(\mathrm{~F}=38.3378 \mathrm{P}=0.0001)$, in 2017( $\mathrm{F}=110.8096 \mathrm{P}<0.01)$, in 
2018( $\mathrm{F}=21.6136 \mathrm{P}=0.0275)$ and in $2019(\mathrm{~F}=35.6517 \mathrm{P}=0.0002)$, but no mensal preference was observed in $2014 \otimes F=4.7018 P>0.05 \rrbracket$,in 2015( $F=10.5878, P=0.4784)$ and in $2020(F=10.1404, P=0.5178)$.(Fig1)

\section{The association between $M$. pneumonia infection and $\mathrm{PM}_{2.5}, \mathrm{PM}_{10}$, temperature and humidity}

There was no correlation between $M$. pneumonia infection and temperature $(R=0.1441 \mathrm{P}>0.05)$ and humidity $(R=0.0060 P \otimes 0.05)(F i g 2,3)$. And PM2.5 $(R=0.09362, P<0.01)$ and $P M 10(R=0.1185, P<0.01)$ negative correlation exists (Fig4,5)

\section{The constituent ratio of case of $M$. pneumonia infection}

There was no difference about constituent ratio of case of M. pneumonia infection between 2014 and 2019( $F=32.34 \mathrm{P}>0.05)$. The Common respiratory diseases of M..pneumonia infection, bronchopneumonia accounts for the highest proportion,followed the exacerbation of asthma and severe pneumonia.But there was difference about constituent ratio of case of M..pneumonia infection in 2020, Compared with other years $(F=159.35 \mathrm{P}<0.01)$, The incidence of severe pneumonia decreased significantly. The Common respiratory diseases of M..pneumonia infection, bronchopneumonia accounts for the highest

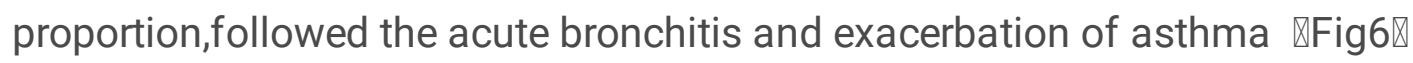

\section{Discussion}

M.pneumonia is a microorganism as bacteria and virus, it can cause pharyngitis, otitis, tracheobronchitis, or bronchopneumonia, transmitted by respiratory droplets. But the positive rate of M. pneumonia is different obviously in different countries, different areas, different ages, different years and different seasonal groups. To provide the more evidence to make a diagnosis and give treatments, it is important to know the characteristic of M. pneumonia in local area .

There was $18.41 \%$ ratio of $M$. pneumonia infection in respiratory tract infection patients in hospital from 2014 to 2020 in Chengdu, It was lower than in other areas in China ${ }^{[5]}$, t was also lower than other countries, such as in Japan ,in Peru ${ }^{[6][7]}$ but it was higher than in Russia where the M. pneumonia was detected about $15,9 \%{ }^{[8]}$ It was believed that geographical factor contributed to the apparent differences of M. pneumonia infections $₫ \mathrm{O}$ course, The different results were affected by various research samples and different detection methods .

The gender distribution in M. pneumonia had different reports ${ }^{[6]}$, which showed the positive percentage of $M$. pneumonia in male children was lower than that of female children. The similar result was reported in other areas in China ${ }^{[9][10]}$. but the reasons for differences in prevalence according to sex are unexplained now, The more studies should be confirmed in the future.

Our data showed a significant association among age groups. Age is an important factor that can affect pathogen distribution, We found the preschoolers and school-age children had the higher incidence rate of M. pneumonia infections which reached $33.98 \%$ and32.98\% . In China, children start live or study in 
crowed because of large population at age of three ,such as in kindergarten,in primary school ,so the $M$. pneumonia is most easily spread among preschoolers and school-age children .(chart1 P凶0.05) ..A study conducted in India which has the same large population also reported the positive rate of $M$. pneumonia infection was $59.5 \%$ in Children ${ }^{[11]}$.At the same time, the immune response ability of organism is improved, and the pathogen can be produced effective response in older children.So the M. pneumonia infections played an important role in this age group. Therefor,lt should pay more attention to improve the learning and living environment of preshoolers and school-age children as conditions allow.

There were different positive percentage of M. pneumonia in different years from 2014 to 2020. It was reported the epidemics of $M$. pneumonia infections occurred at intervals of 3 to 7 years ${ }^{[12]}$ There was a general upward trend in volatility in recent seven years in our study. It seemed the $M$. pneumonia infection epidemic time was 2017and 2019 in Chengdu, respectively 26.59\%and 28.18\%.it was the similar report that there was a outbreak of M. pneumonia in Finland in $2017^{\text {[13] }}$. To our surprise, there was a lower incidence of $M$. pneumonia infection in 2020, when 2019 coronavirus diseases (COVID-19) spreaded in China. What's more, the incidence of M. pneumonia infection was little seasonal variation in 2020.Since the prevention and control of the COVID-19 epidemic from January 2020,The prevention measures had effectively controlled the infection rate of M. pneumonia. Although M.pneumonia infection can happen at any time of year, it was most common throughout April to May and September to October in 2016,2017,2018 and 2019 in Chengdu (Figure1 Pख0.05), it was different from other areas ${ }^{[14]}$ ${ }^{[15]}$.Though,Our study demonstrated that there was seasonal variation in the incidence of $M$. pneumonia infection. Interestingly,we also found that there was a relatively lower rate of $M$. pneumonia infection in winter vacation (February)and summer vacation(July,August) every year in Chengdu. We Suspicious the physical changes after the vacation, and the crowded environment in school also play a important role in M. pneumonia infection in children.So it is important for doctors not only to know the character of sustainability and aperiodic spreading about $M$. pneumonia in local area, to do regular monitoring of $M$. pneumonia forever,but also to enhance the education how to avoid the M. pneumonia infection in school.

In this study ,We uniquely explored whether there was an association between $\boldsymbol{M}$. pneumonia infection and $\mathrm{PM}_{2.5}$ ( The fine particulate matter in the air $<2.5 \mathrm{um}$ ) $\mathrm{PM}_{10}$

( The fine particulate matter in the air $<10 \mathrm{um}$ ), temperature and humidity in Chengdu. Some cohort studies had identified consistent associations between ambient $\mathrm{PM}_{2.5}$ and cardiorespiratory morbidity ${ }^{[16]}$.Our study showed there was negative correlation between the positive rate of $M$. pneumonia infection and $\mathrm{PM}_{2.5}, \mathrm{PM}_{10}$. In fact, There was serious air pollution in Chengdu in 2014and 2015. It had been improvement after making some progress in environmental protection to 2020 . Air pollution increased the number respiratory tract infection cases, but the pathogeny may be not the $M$. pneumonia,so the positive rate of M. pneumonia infection was relatively low in 2014 and 2015. There was no associated between $M$. pneumonia infection with the average temperature and humidity of the climate, it was also different from other opinion ${ }^{[17], T h e ~ t e m p e r a t u r e ~ a n d ~ h u m i d i t y ~ o f ~ t h e ~ c l i m a t e ~ m a y b e ~}$ affect the growth of M.pneumonia ,but during the study, we only analyzed the infection of 
M.pneumonia,not carriage of M. pneumonia .After all,there was high rates of healthy children carry $M$. pneumonia $^{[18]}$.

In the hospitalized children who were infected with M. pneumonia between 2014 and 2019, The cases of bronchopneumonia was the most common,followed by acute exacerbation of asthma and severe pneumonia. it was the similar opinion that the M.pneumonia infection played a key role in CAP (community-acquried pneumonia) in children ${ }^{[19][20] .}$. But other studies showed the highest $M$. pneumonia positive rate for children with acute bronchitis ${ }^{[21]}$. The different results were may be affected by the object being investigated who came from the inpatient children only, not outpatient children in our study . Previous studies showed $M$. pneumonia may act a part in the onset of asthma in predisposed children and could cause recurrent wheezing ${ }^{[22]}$. our study also confirmed that the infection with $M$. pneumonia was frequently correlated with exacerbation of asthma in Children ${ }^{[23]}$, so it maybe suggested empirical treatments to M. pneumonia infection in the children with exacerbation of Asthma, before knowing the exact pathogen. Though the M. pneumonia pneumonia (MPP) is typically mild and self-limiting, but severe M. pneumonia pneumonia (SMPP), including severe pulmonary complications( such as obliterative bronchitis,bronchiectasis and necrotizing pneumonia (NP)) have increased in China ${ }^{\text {[24]. In our }}$ study, we also found the cases of severe pneumonia was common too in the children inpatients who were infected with M. pneumonia,what's more, Lobar pneumonia increased in 2017,2018 and 2019. But the the rate of severe pneumonia had significantly reduced in 2020. We suspected whether the COIVD-19 maybe weaken the virulence of M. pneumonia, The more studies should be confirmed in the future. However, in the M. pneumonia, epidemic time,we should aware of the possibility of severe lung disease.

In conclusions, through retrospective analysis of the M. pneumonia results in Chengdu from 2014 to 2020,we know the characteristic about M. pneumonia in local area. It can help doctors to make clinical diagnosis and treatments and prevention of respiratory tract infection reasonably. Comparing the different characteristic of M. pneumonia in 2020 with in other years, we found the prevention strategies such as health education,washing hands,wearing mask can also control the epidemic of $M$. pneumonia.But there were several limitations to our study .First, this study do not analyze the characteristic of outpatients of $M$. pneumonia infection. Second,our testing did not include analyzing the patients with the single M. pneumonia infection or co-infection with other pathogen.

\section{References}

[1] Waites KB, Xiao L, Liu Y, et al. Mycoplasma pneumoniae from the Respiratory Tract and Beyond[J]. Clin Microbiol Rev, 2017, 30(3): 747-809. doi: 10.1128/cmr.00114-16

[2] Rogozinski LE, Alverson BK, Biondi EA. Diagnosis and treatment of Mycoplasma pneumoniae in children[J]. Minerva Pediatr, 2017, 69(2): 156-160. doi: 10.23736/s0026-4946.16.04866-0

[3] Gao LW, Yin J, Hu YH, et al. The epidemiology of paediatric Mycoplasma pneumoniae pneumonia in North China: 2006 to 2016[J]. Epidemiol Infect, 2019, 147: e192. doi: 10.1017/s0950268819000839 
[4] Expert Committee on Rational Use of Medicines for Children Pharmaceutical Group NHaPC. [Expert consensus on laboratory diagnostics and clinical practice of Mycoplasma pneumoniae infection in children in China (2019)][J]. Zhonghua Er Ke Za Zhi, 2020, 58(5): 366-373. doi: 10.3760/cma.j.cn11214020200304-00176

[5] Chen K, Jia R, Li L, et al. The aetiology of community associated pneumonia in children in Nanjing, China and aetiological patterns associated with age and season[J]. BMC Public Health, 2015, 15: 113. doi: 10.1186/s12889-015-1422-1

[6] Oishi T, Fukuda Y, Wakabayashi S, et al. Low prevalence of Chlamydia pneumoniae infections during the Mycoplasma pneumoniae epidemic season: Results of nationwide surveillance in Japan[J]. J Infect Chemother, 2020, 26(11): 1116-1121. doi: 10.1016/j.jiac.2020.04.015

[7] Del Valle-Mendoza J, Orellana-Peralta F, Marcelo-Rodríguez A, et al. High Prevalence of Mycoplasma pneumoniae and Chlamydia pneumoniae in Children with Acute Respiratory Infections from Lima, Peru[J]. PLoS One, 2017, 12(1): e0170787. doi: 10.1371/journal.pone.0170787

[8] Voronina EN, Gordukova MA, Turina IE, et al. Molecular characterization of Mycoplasma pneumoniae infections in Moscow from 2015 to 2018[J]. Eur J Clin Microbiol Infect Dis, 2020, 39(2): 257-263. doi: $10.1007 /$ s10096-019-03717-6

[9] Yan C SH, Zhao HQ, et al. Epidemiological characteristics of Mycoplasma pneumoniae infection in hospitalized children in Beijing: 10-year retrospective analysis[J]. Chin J Appl Clin Pediatr, 2019.

[10] Zhang XX JW, Gu WJ, et al. Epidemiological analysis of Myeoplasma pneumoniae infection in children with respiratory tract diseases in Suzhou area from 2005 to 2014[J]. Clin J Infect Dia 2015.[11] Kumar S, Garg IB, Sethi GR. Mycoplasma pneumoniae in Community-Acquired Lower Respiratory Tract Infections[J]. Indian J Pediatr, 2018, 85(6): 415-419. doi: 10.1007/s12098-017-2580-1

[12] Lenglet A, Herrador Z, Magiorakos AP, et al. Surveillance status and recent data for Mycoplasma pneumoniae infections in the European Union and European Economic Area, January 2012[J]. Euro Surveill, 2012, 17(5). doi: 10.2807/ese.17.05.20075-en

[13] Kurkela S, Puolakkainen M, Hokynar K, et al. Mycoplasma pneumoniae outbreak, Southeastern Finland, 2017-2018: molecular epidemiology and laboratory diagnostic lessons[J]. Eur J Clin Microbiol Infect Dis, 2019, 38(10): 1867-1871. doi: 10.1007/s10096-019-03619-7

[14] Ma YJ, Wang SM, Cho YH, et al. Clinical and epidemiological characteristics in children with community-acquired mycoplasma pneumonia in Taiwan: A nationwide surveillance[J]. J Microbiol Immunol Infect, 2015, 48(6): 632-638. doi: 10.1016/j.jmii.2014.08.003

[15] Zhao H, Li S, Cao L, et al. Surveillance of Mycoplasma pneumoniae infection among children in Beijing from 2007 to 2012[J]. Chin Med J (Engl), 2014, 127(7): 1244-1248. 
[16] Hou W, Xu X, Lei Y, et al. The role of the PM2.5-associated metals in pathogenesis of child Mycoplasma Pneumoniae infections: a systematic review[J]. Environ Sci Pollut Res Int, 2016, 23(11): 10604-10614. doi: 10.1007/s11356-016-6535-2

[17] Onozuka D, Hashizume M, Hagihara A. Impact of weather factors on Mycoplasma pneumoniae pneumonia[J]. Thorax, 2009, 64(6): 507-511. doi: 10.1136/thx.2008.111237

[18] Meyer Sauteur PM, Unger WW, Nadal D, et al. Infection with and Carriage of Mycoplasma pneumoniae in Children[J]. Front Microbiol, 2016, 7: 329. doi: 10.3389/fmicb.2016.00329

[19] Chi H, Huang YC, Liu CC, et al. Characteristics and etiology of hospitalized pediatric communityacquired pneumonia in Taiwan[J]. J Formos Med Assoc, 2020, 119(10): 1490-1499. doi: 10.1016/j.jfma.2020.07.014

[20] Wang Z, Ji Y, Zhang J, et al. Investigation on Atypical Pathogens related with Community Acquired Pneumonia and the Factors Associated with Mycoplasma Pneumoniae Infection in Jiangsu, China[J]. Clin Lab, 2020, 66(6). doi: 10.7754/Clin.Lab.2019.191036

[21] Vervloet LA, Marguet C, Camargos PA. Infection by Mycoplasma pneumoniae and its importance as an etiological agent in childhood community-acquired pneumonias[J]. Braz J Infect Dis, 2007, 11(5): 507514. doi: $10.1590 /$ s1413-86702007000500012

[22] Chen J, Ji F, Yin Y, Yuan S.Time to Mycoplasma Pneumoniae RNA Clearance for Wheezy vs. NonWheezy Young Children with Community-Acquired Pneumonia.J Trop Pediatr,2020 Dec 4;fmaa109. doi: 10.1093/tropej/fmaa109

[23] Kumar S, Roy RD, Sethi GR, et al. Mycoplasma pneumoniae infection and asthma in children[J]. Trop Doct, 2019, 49(2): 117-119. doi: 10.1177/0049475518816591

[24] Zheng B, Zhao J, Cao L. The clinical characteristics and risk factors for necrotizing pneumonia caused by Mycoplasma pneumoniae in children[J]. BMC Infect Dis, 2020, 20(1): 391. doi: $10.1186 / \mathrm{s} 12879-020-05110-7$

\section{Figures}




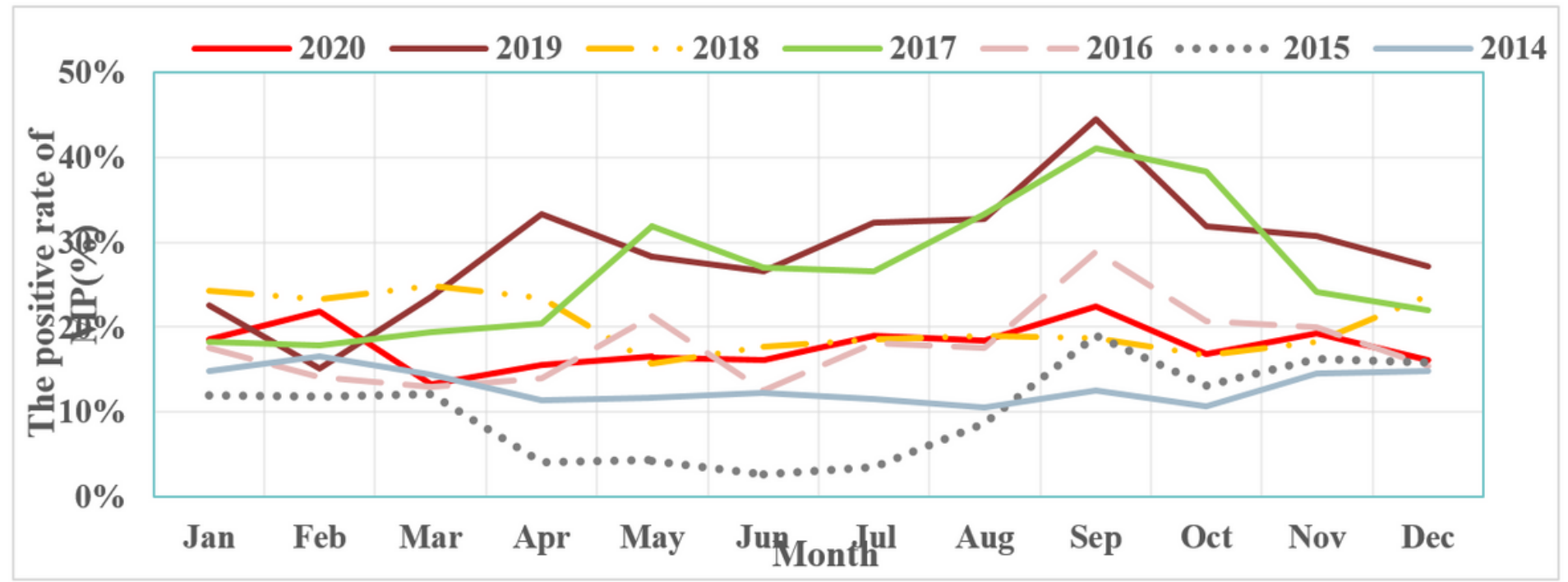

Figure 1

The seasonal incidence rates of M. pneumonia from 2014 to 2020.

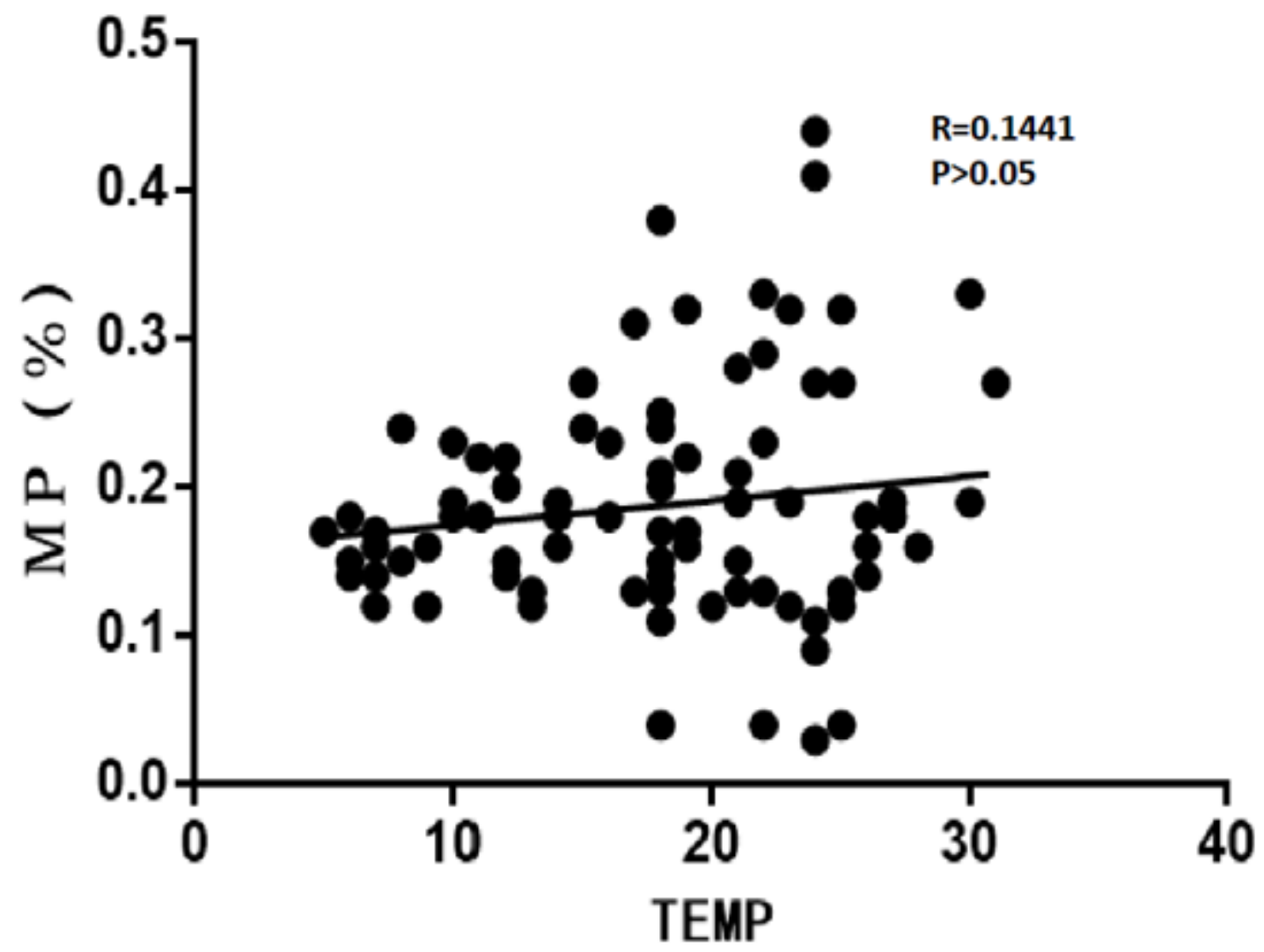

Figure 2

The correlation analysis between temperature, humidity with the positive rate of $\mathrm{M}$. pneumonia infection 


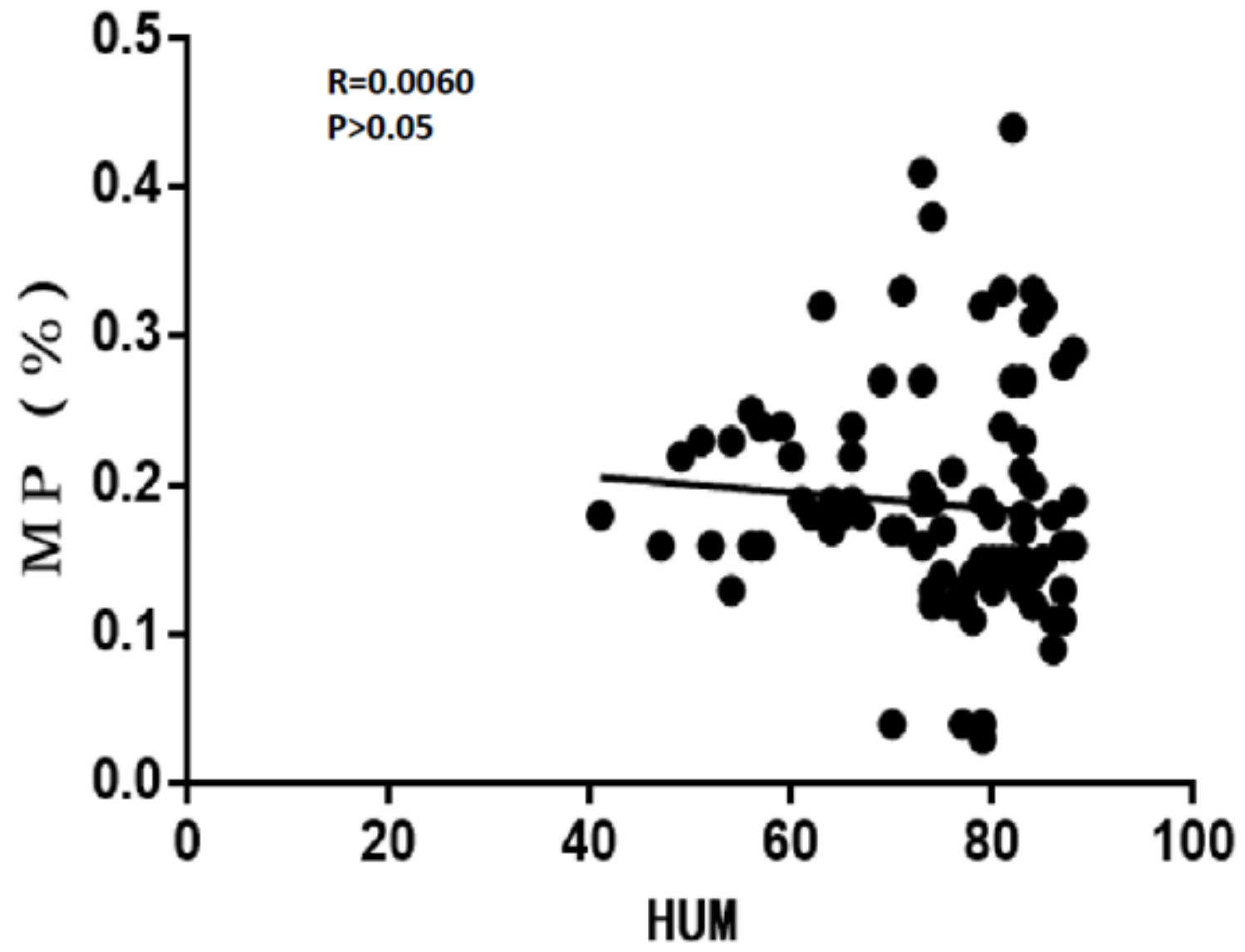

Figure 3

The correlation analysis between temperature, humidity with the positive rate of $\mathrm{M}$. pneumonia infection 


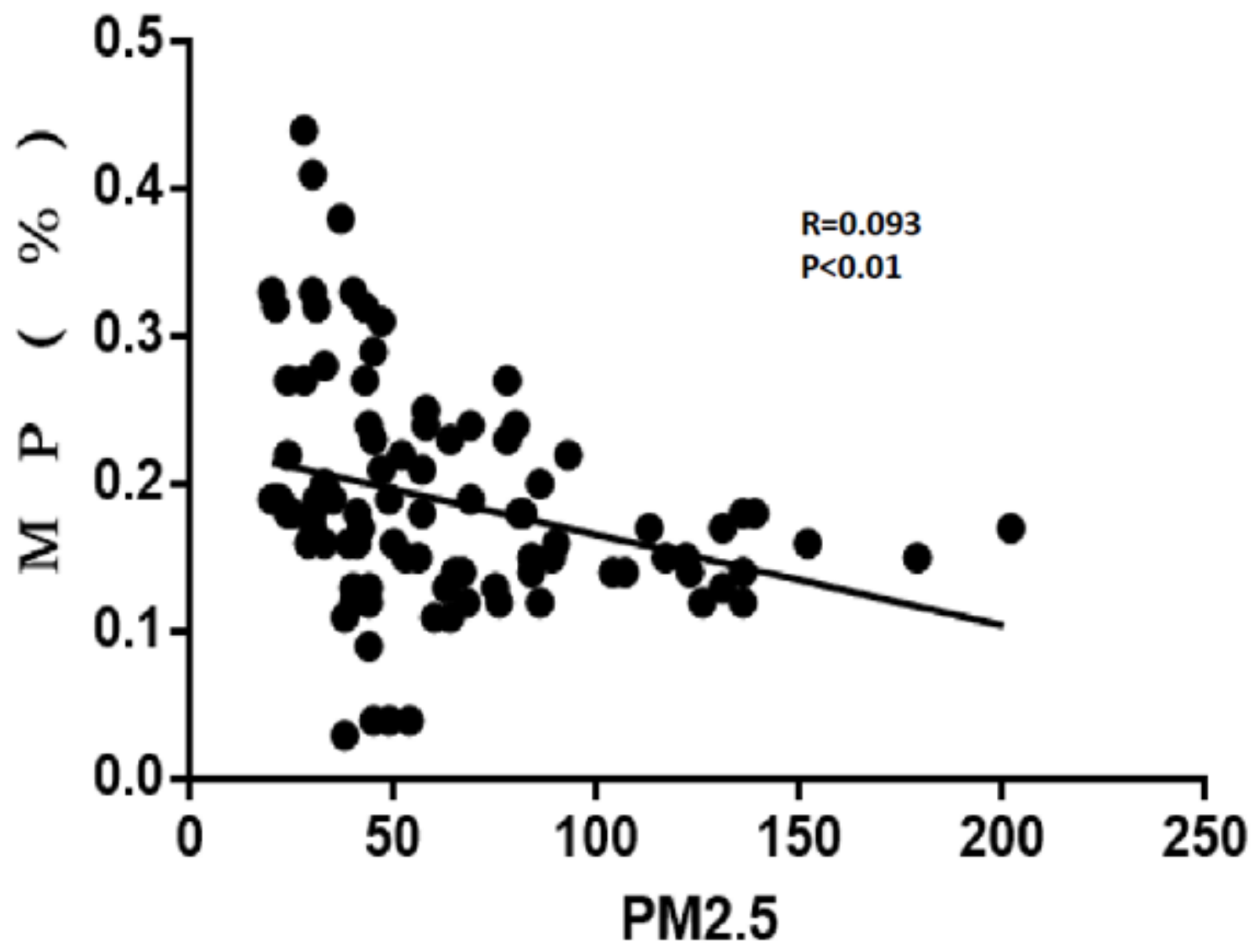

Figure 4

The correlation analysis between PM2.5 ,PM10 with the rate of M. pneumonia infection 


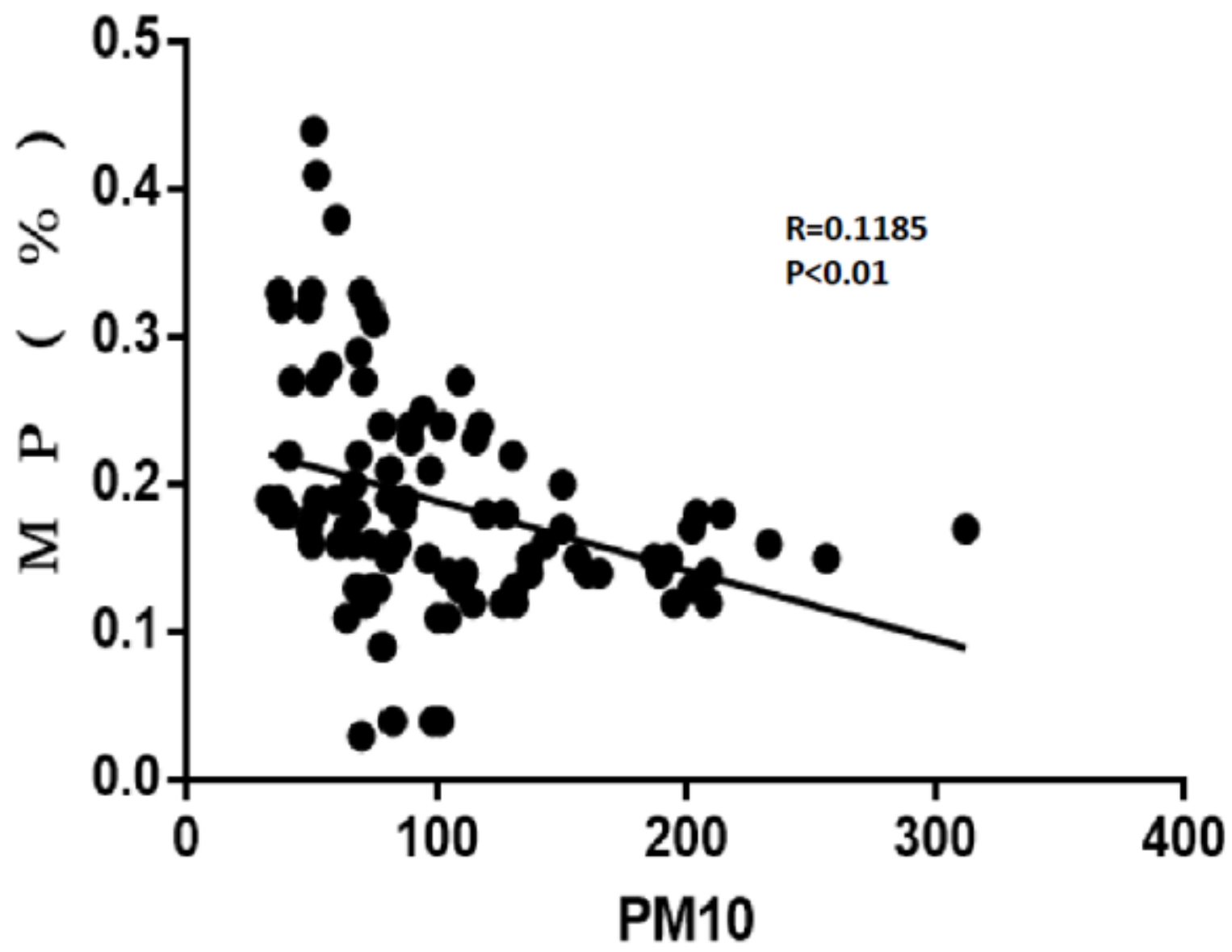

Figure 5

The correlation analysis between PM2.5 ,PM10 with the rate of M. pneumonia infection 


\begin{tabular}{|c|c|c|c|}
\hline$\$$ Bronchopneumonia & OAsthma & \# Severe pneumonia & Lobar pneumonia \\
\hline$=$ ILD & - Acute bronchitis & ¿Capillary bronchitis & Ehronic cough \\
\hline Suppurative tonsilitis & - Asthmatoid bronchitis & - Sepsis & - Others \\
\hline
\end{tabular}

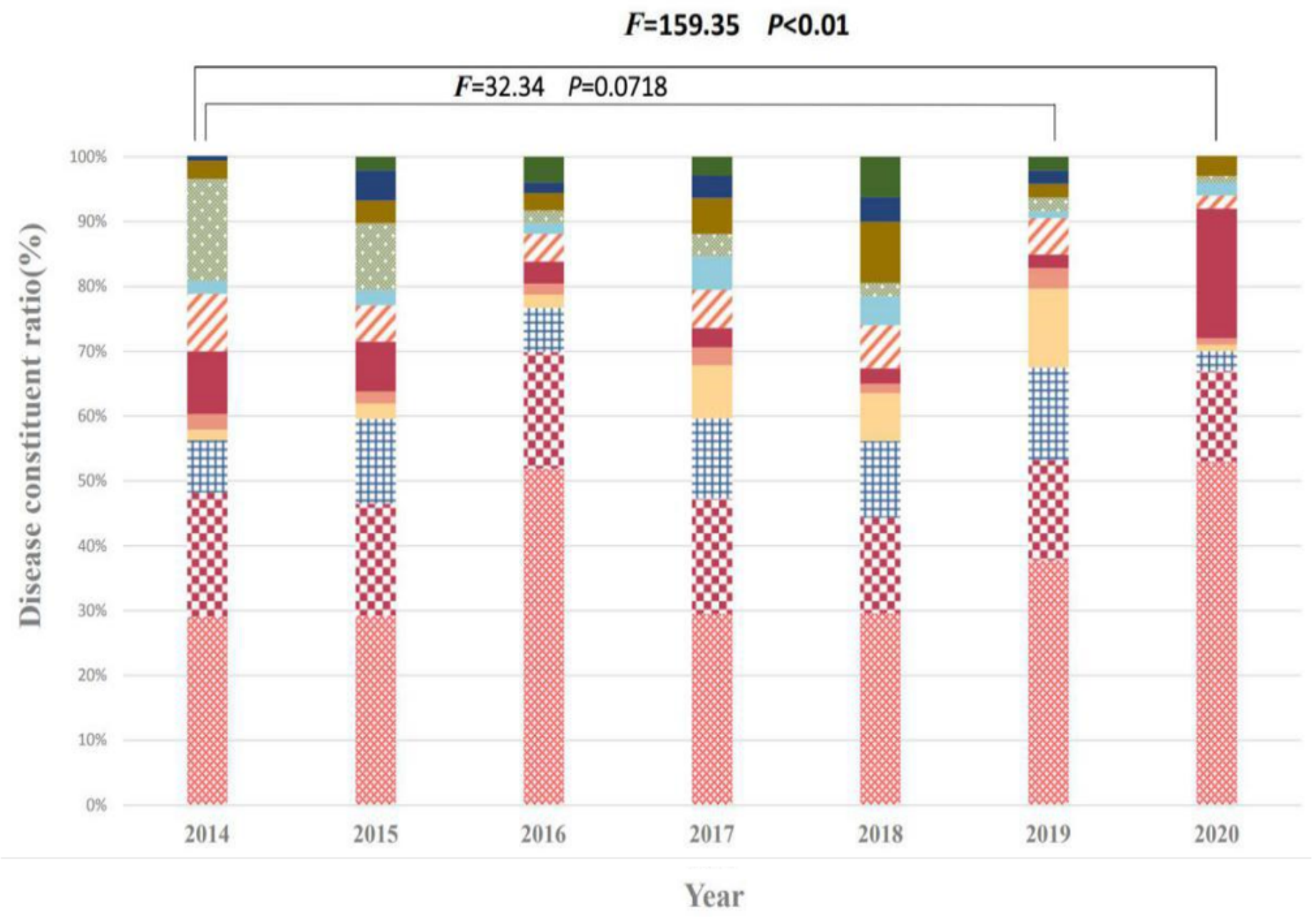

Asthma :exacerbation of asthma ILD: Interstitial lung Disease

\section{Figure 6}

The constituent ratio of case of M. pneumonia infection 